Nota científica

\title{
Registros adicionales de la serpiente caracolera occidental Tropidodipsas annulifera (Serpentes: Dipsadidae) en el estado de Jalisco, México
}

\author{
Additional records of the Western Snail-eating snake Tropidodipsas annulifera \\ (Serpentes: Dipsadidae) in the state of Jalisco, Mexico
Iván Trinidad Ahumada-Carrillo ${ }^{\mathrm{a}, *}$, Ginny Nicole Weatherman ${ }^{\mathrm{b}}$ y Octavio Vázquez-Huizar ${ }^{\mathrm{a}}$
${ }^{a}$ Centro Universitario de Ciencias Biológicas y Agropecuarias, Zapopan, Jalisco, México
${ }^{\mathrm{b}}$ Department of Ecology \& Evolutionary Biology, University of Kansas, 2041 Haworth Hall, 1200 Sunnyside Avenue, Lawrence, Kansas 66045, EUA \\ Recibido el 30 de julio de 2015; aceptado el 21 de octubre de 2015 \\ Disponible en Internet el 2 de marzo de 2016
}

\begin{abstract}
Resumen
Con base en 2 ejemplares, documentamos la presencia de Tropidodipsas annulifera en los municipios de Chapala y Zapopan, Jalisco, México. Esta especie había sido registrada previamente en Jalisco solo con 9 ejemplares. En este trabajo extendemos la distribución conocida en el estado. Adicionalmente, se presentan nuevos datos sobre las características morfológicas de la especie.

Derechos Reservados (C) 2016 Universidad Nacional Autónoma de México, Instituto de Biología. Este es un artículo de acceso abierto distribuido bajo los términos de la Licencia Creative Commons CC BY-NC-ND 4.0.
\end{abstract}

Palabras clave: Tropidodipsas annulifera; Distribución; Chapala; Zapopan

\begin{abstract} we present new data on morphological characters of the species. Creative Commons CC License BY-NC-ND 4.0.

Keywords: Tropidodipsas annulifera; Distribution; Chapala; Zapopan
\end{abstract}

Based on 2 specimens, we documented the occurrence of Tropidodipsas annulifera in the municipalities of Chapala and Zapopan, Jalisco, Mexico. This species was previously recorded in Jalisco only by 9 specimens. Herein we extend the known distribution in the state. Additionally,

All Rights Reserved (C) 2016 Universidad Nacional Autónoma de México, Instituto de Biología. This is an open access item distributed under the

El género Tropidodipsas (Günther, 1858) está compuesto por 7 especies actualmente reconocidas: Tropidodipsas annulifera, T. fasciata, T. fischeri, T. philippii, T. repleta, T. sartorii y T. zweifeli (Smith, Lemos-Espinal, Hartman y Chiszar, 2005; Wallach, 1995). Este género se encuentra cercanamente relacionado con el género Sibon, en donde Kofron (1985) incluyó todas las especies del género Tropidodipsas. Wallach (1995) mostró evidencia para revalidar el género Tropidodipsas, y determinó que el estatus de $T$. fischeri permanece aún sin resolver. Todas

\footnotetext{
* Autor para correspondencia.

Correo electrónico: lepidus320@hotmail.com (I.T. Ahumada-Carrillo).

La revisión por pares es responsabilidad de la Universidad Nacional Autónoma de México.
}

las especies de Tropidodipsas son de hábitos nocturnos, principalmente terrestres, y presentan una dieta especializada en gasterópodos (Köhler, 2003). Se encuentran distribuidas en altitudes que van de 0 a 3,000 m snm (Kofron, 1988; Köhler, 2003; Muñoz-Alonso, 2007).

La serpiente caracolera occidental (Tropidodipsas annulifera) se distingue de las otras especies del género, por la combinación de las siguientes características: en vida, el cuerpo presenta una coloración negra con bandas o anillos de color blanco o amarillo (3-14); escamas con fosetas apicales ausentes; las escamas alrededor del cuerpo se encuentran dispuestas en la siguiente fórmula 15-15-15, generalmente quilladas dorsalmente; número de escamas ventrales de 139-155 - 142-155 en hembras y 139-154 en machos_-, subcaudales de 38-52 - 38-52 


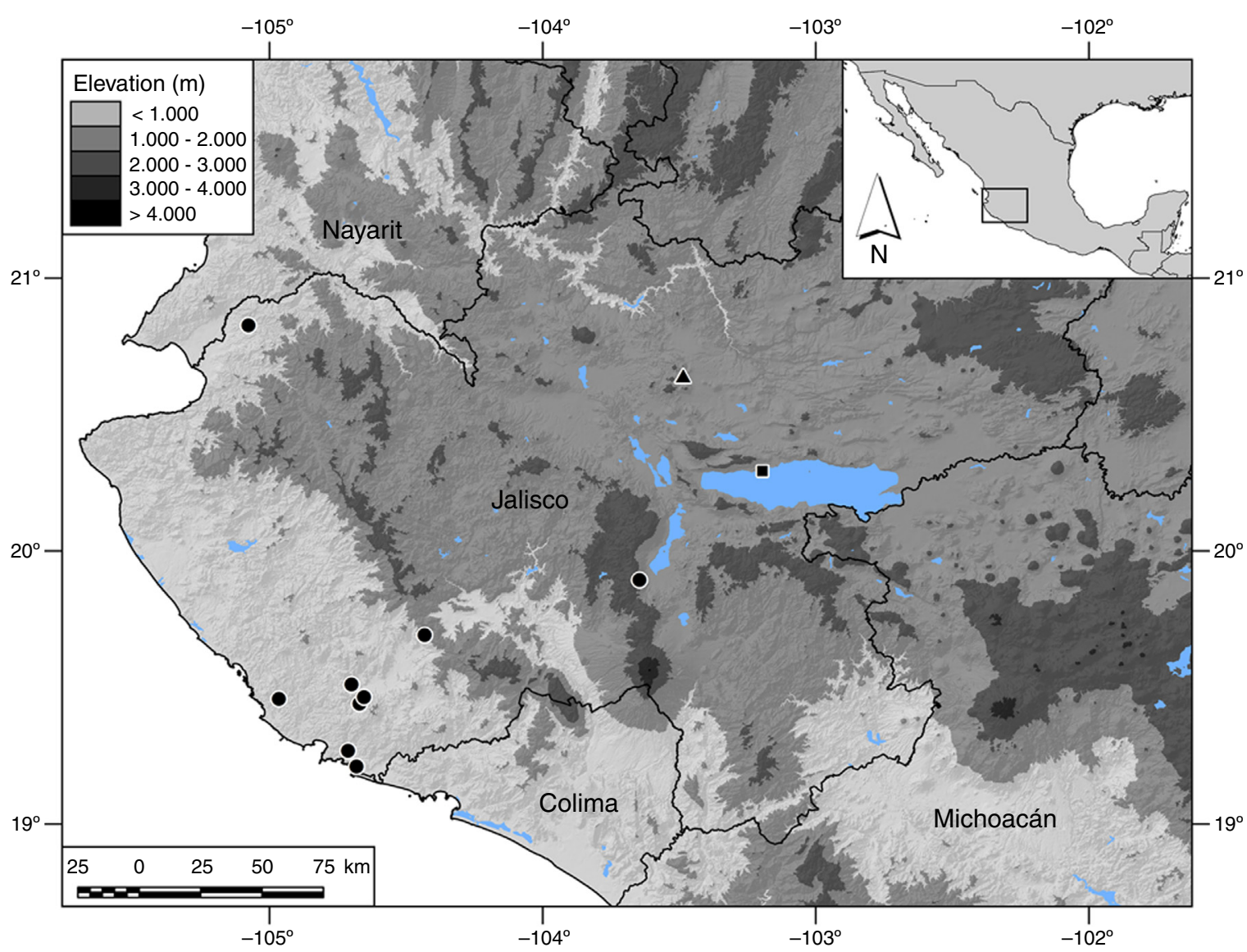

Figura 1. Mapa de la distribución geográfica de Tropidodipsas annulifera en el estado de Jalisco. Los círculos negros representan los registros previos; el triángulo, el ejemplar UTADC-8550 y el cuadro, el ejemplar UTADC-8551.

en hembras y $42-52$ en machos-; un par de prefrontales; infralabiales 7-9; supralabiales 6-7; escama anal simple, y dientes maxilares de 8-11 (Kofron, 1988).

Tropidodipsas annulifera es una especie endémica a México, y se distribuye a través de la costa del Pacífico en los estados de Sinaloa, Nayarit, Jalisco, Colima, Michoacán, Guerrero y en la isla María Madre (Kofron, 1988; Reyes-Velasco, Grünwald, \& Jones, 2008; Reyes-Velasco, Hermosillo-López, Grünwald y Ávila- López, 2009; Scott, 1967). Habita principalmente los ambientes tropicales de la costa del Pacífico y las sierras adyacentes en elevaciones que van de 0 a 1,909 m snm (Kofron, 1988) (fig. 1). En la Norma Oficial Mexicana (Nom-059-Semarnat2010) se encuentra en la categoría de sujeta a protección especial ( $\mathrm{Pr}$ ) y en la lista roja de la Unión Internacional para la Conservación de la Naturaleza (UICN) en la categoría de preocupación menor (LC, por sus siglas en inglés) (Ponce-Campos y García-Aguayo, 2007; Semarnat, 2010). Adicionalmente, Wilson, Mata-Silva y Johnson (2013) ubican a esta especie en la categoría de vulnerabilidad media en el environmental vulnerability score (EVS).

Actualmente en Jalisco existen registros de esta especie para 9 localidades: $70 \mathrm{~km}$ al suroeste de Autlán de Navarro (Scott, 1967); Barra de Navidad; $113 \mathrm{~km}$ al suroeste de Autlán de Navarro; $32.2 \mathrm{~km}$ al sur de La Huerta; $6.4 \mathrm{~km}$ al oeste de La Huerta; $40.2 \mathrm{~km}$ al suroeste del río San Pedro sobre la carretera 46; $18 \mathrm{~km}$ al sureste de Tapalpa (Kofron, 1988); Las Palmas, en Puerto Vallarta (Myska, 2014), y la región de Chamela (García y Valtierra-Azotla, 1996). En el presente trabajo se registran 2 localidades adicionales (fig. 1). Municipio de Chapala: cerro del Tepehua (UTADC-8551; 20 $17^{\prime} 34.41^{\prime \prime}$ N, 103 $11^{\prime} 42.83^{\prime \prime}$ O; WGS 84; 1,595 m snm), 20/VIII/2014; el ejemplar se encontró desplazándose en la noche en las afueras de una casa habitación, misma que colinda directamente con un relicto de bosque tropical caducifolio (fig. 2A). Municipio de Zapopan: Área Natural Protegida (ANP) Bosque La Prima-

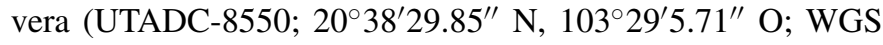
84; $1,770 \mathrm{~m} \mathrm{snm}$ ), 20/VIII/2014; el ejemplar se encontró bajo un tronco en descomposición al interior de un bosque de encino (fig. 2B).

Ambos ejemplares se midieron en campo, se tomaron los datos morfológicos, sexo y coloración (tabla 1). El ejemplar de Chapala (UTADC-8551) presentó 36 escamas subcaudales, número inferior a los registrados en hembras por Kofron (1988), así como un número superior de escamas supralabiales en el lado derecho (tabla 1). Los ejemplares se fotografiaron y liberaron en el sitio de recolecta. Las fotografías de los ejemplares registrados fueron depositadas en la University of Texas at Arlington-Digital Collection (UTADC). Estos registros representan una ampliación en su distribución a una distancia aproximada de $65 \mathrm{~km}$ (Chapala) y $86 \mathrm{~km}$ (ANP Bosque La 
Tabla 1

Características morfométricas y morfológicas de los 2 nuevos ejemplares de Tropidodipsas annulifera registrados en Jalisco.

\begin{tabular}{|c|c|c|c|c|c|c|c|c|c|c|}
\hline Ejemplar & $\mathrm{EV}$ & ES & EI & SP & LT & LHC & $\mathrm{LC}$ & $\mathrm{ACC}$ & ACL & Sexo \\
\hline UTADC-8550 & 149 & 44 & $7-7$ & $6-6$ & $49.7 \mathrm{~cm}$ & $41 \mathrm{~cm}$ & $8.7 \mathrm{~cm}$ & 12 & 5 & $\mathrm{H}$ \\
\hline UTADC-8551 & 146 & 36 & $7-7$ & $7-8$ & $26.9 \mathrm{~cm}$ & $20.5 \mathrm{~cm}$ & $6.4 \mathrm{~cm}$ & 14 & 2 & $\mathrm{H}$ \\
\hline
\end{tabular}

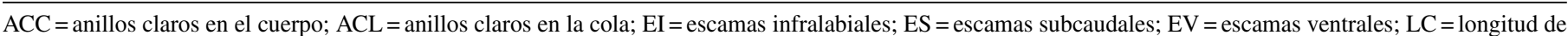
la cola; $\mathrm{LHC}=$ longitud hocico-cloaca; $\mathrm{LT}=$ longitud total; $\mathrm{SP}=$ escamas supralabiales.
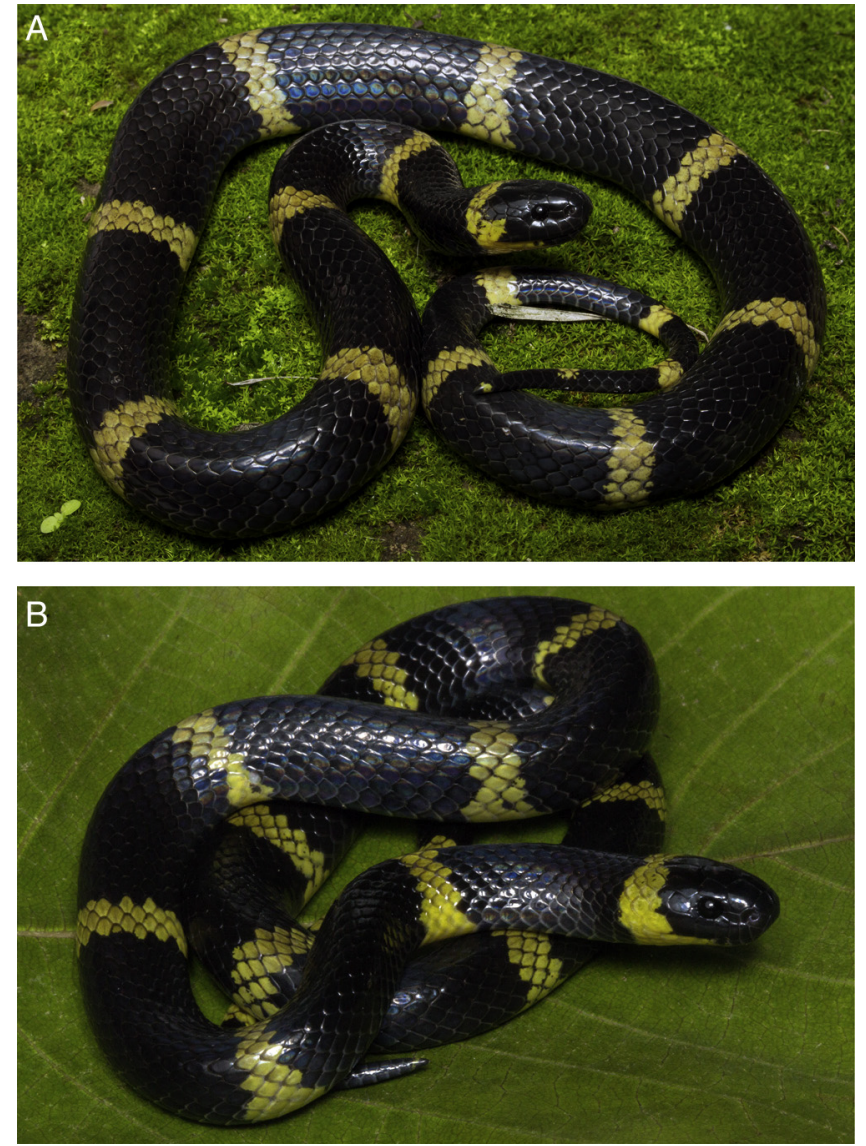

Figura 2. A) Ejemplar registrado en el Bosque La Primavera, municipio de Zapopan (UTADC-8550). B) Ejemplar proveniente del cerro el Tepehua, municipio de Chapala (UTADC-8551).

Primavera), ambas en dirección noreste con respecto al registro más cercano ubicado a $18 \mathrm{~km}$ al sureste de Tapalpa. El ejemplar UTADC-8550 representa un primer registro adicional para la herpetofauna previamente conocida en el ANP Bosque La Primavera, ya que no había sido registrada anteriormente en el trabajo de Reyna-Bustos, Ahumada-Carrillo y Vázquez-Huizar (2007). Asimismo, se amplía el conocimiento sobre la variación morfológica de la especie.

Agradecemos a Christian Chávez por proveernos el ejemplar UTADC-8550, a Rubén A. Carbajal-Márquez por sus comentarios y sugerencias en la versión previa del escrito, y a José
Carlos Arenas-Monroy por la elaboración del mapa. Así como al Dr. José Rogelio Cedeño-Vázquez y a 2 revisores anónimos por los comentarios aportados para mejorar la versión final.

\section{Referencias}

García, A. y Valtierra-Azotla, M. (1996). Sibon annuliferus (Western Snaileating Snake). Herpetological Review, 27, 89.

Günther, A. (1858). Catalogue of Colubrine snakes in the Collection of the British Museum. Londres: Trustees of the British Museum.

Kofron, C. P. (1985). Systematics of neotropical gastropod-eating snake genera Tropidodipsas and Sibon. Journal of Herpetology, 19, 84-92.

Kofron, C. P. (1988). Systematics of neotropical gastropod-eating snakes: the sartorii group of the genus Sibon. Amphibia-Reptilia, 9, 145-168.

Köhler, G. (2003). Reptiles of Central America. Offenbach: Herpeton-Verlag Elke Köhler.

Muñoz-Alonso, A. (2007). Sibon fischeri. The IUCN Red list of threatened species. Versión 2014. Recuperado el 21 de julio de 2014 de: http://www.iucnredlist.org

Myska, P. (2014). Viva natura: field guide to the amphibians, reptiles, birds and mammals of Western Mexico/Guía de campo de anfibios, reptiles, aves y mamíferos de México occidental. Puerto Vallarta, Jalisco: Viva Natura/ Conabio.

Ponce-Campos, P. y García-Aguayo, A. (2007). Tropidodipsas annulifera. The IUCN Red list of threatened species. Versión 2014.3. Recuperado el 25 de noviembre de 2014 de: http://www.iucnredlist.org

Reyes-Velasco, J., Grünwald, C. I. y Jones, J. (2008). Tropidodipsas annulifera (Western snailsucker). Herpetological Review, 39, 487

Reyes-Velasco, J., Hermosillo-López, I. A., Grünwald, C. I. y Ávila- López, O. A. (2009). New state records for amphibians and reptiles from Colima, Mexico. Herpetological Review, 40, 117-120.

Reyna-Bustos, O. F., Ahumada-Carrillo, I. T. y Vázquez-Huizar, O. (2007). Anfibios y reptiles del Bosque La Primavera: guía ilustrada. Guadalajara, Jalisco: Universidad de Guadalajara, Gobierno del Estado de Jalisco.

Scott, N. J., Jr. (1967). The colubrid snake, Tropidodipsas annulifera, with reference to the status of Geatractus, Exelencophis, Chersodromus annulatus, and Tropidodipsas malacodryas. Copeia, 1967, 280-287.

Semarnat (Secretaría de Medio Ambiente y Recursos Naturales) (2010). Norma Oficial Mexicana NOM-059-SEMARNAT-2010, Protección ambientalEspecies nativas de México de flora y fauna silvestres-Categorías de riesgo y especificaciones para su inclusión, exclusión o cambio-Lista de especies en riesgo. Diario Oficial de la Federación. 30 de diciembre de 2010, Segunda Sección. México.

Smith, H. M., Lemos-Espinal, J. A., Hartman, D. y Chiszar, D. (2005). A new species of Tropidodipsas (Serpentes: Colubridae) from Sonora, Mexico. Bulletin of the Maryland Herpetological Society, 41, 39-41.

Wallach, V. (1995). Revalidation of the genus Tropidodipsas Günther, with notes on the Dipsadini and Nothopsini (Serpentes: Colubridae). Journal of Herpetology, 29, 476-481.

Wilson, L. D., Mata-Silva, V. y Johnson, J. D. (2013). A conservation reassessment of the reptiles of Mexico based on the EVS measure. Amphibian and Reptile Conservation, 7, 1-47. 